\title{
A Boundary Sensor Control for a Hyper-redundant Arm
}

\author{
Mircea Ivanescu ${ }^{1}$, Dorian Cojocaru ${ }^{1}$, Nicu Bizdoaca ${ }^{1}$, Mihaela Florescu ${ }^{1}$, \\ Nirvana Popescu ${ }^{2}$, Decebal Popescu ${ }^{2}$, Sorin Dumitru ${ }^{1}$ \\ ${ }^{1}$ Department of Mechatronics, University of Craiova, \\ 13, Al. I. Cuza Street, Craiova, 200585, Romania \\ ivanescu@robotics.ucv.ro \\ 2 Computer Department, University Politechnica, Bucharest \\ 313, Splaiul Independenței, Bucharest, Romania \\ nirvana.popescu@cs.pub.ro
}

\begin{abstract}
The paper treats the control problem of a class of hyper-redundant robots with continuum elements with sensors placed on the terminal points of the arm segments. The dynamic model of the arm is presented. The measuring system is based on the sensors placed at the several sub-regions of the arm. A full state control is introduced and an observer is proposed in order to reconstruct the state. A control algorithm is generated by the back-stepping method.
\end{abstract}

\section{Introduction}

The paper treats the control problem of a class of hyper-redundant robots with continuum elements. The control of these systems is very complex and a great number of researchers have tried to offer solutions. In [2, 3], Gravagne analyzed the kinematic models. Important results were obtained by Chirikjian and Burdick [4], which laid the foundations for the kinematical theory of hyper-redundant robots. Their results are based on a "backbone curve" that captures the robot's macroscopic geometric features. Mochiyama has also investigated the problem of controlling the shape of an HDOF rigid - link robot with twodegree-of-freedom joints using spatial curves [5]. In other papers [6,7], several technological solutions for actuators used in hyperredundant structures are presented and conventional control systems are introduced. In [8] control problem of a class that performs the grasping function by coiling is discussed. A frequential stability criterion for the grasping control problem is proposed in $[9,10,11]$.

In this paper, control problem of continuum arms with boundary sensors is treated. Standard feedback control design assumes full-state feedback with measurements of the entire state. Practical constraints require a boundary placement of the sensors. In this case, the development of the state-feedback controllers needs to design state observers.

The paper is organized as follows: section 2 presents technological and theoretical preliminaries, section 3 studies the dynamic model, and section 4 presents the control by boundary observer.

\section{Technological System}

The hyper-redundant technological models are complex structures that operate in 3D space, but the control laws of the elements can be infer from the planar models. For this reason, the model discussed in this paper is a 2D model.

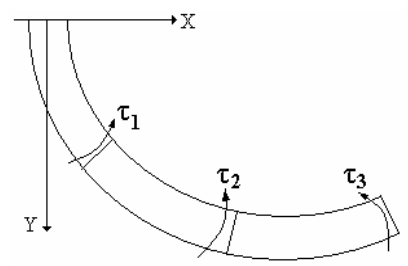

Figure 1. A hyper-redundant arm

The technological model basis is presented in Figure 1. It consists of a number $(\mathrm{N})$ of continuum segments, each segment having a layer structure that ensures the flexibility, the driving and position measuring (Figure 2).

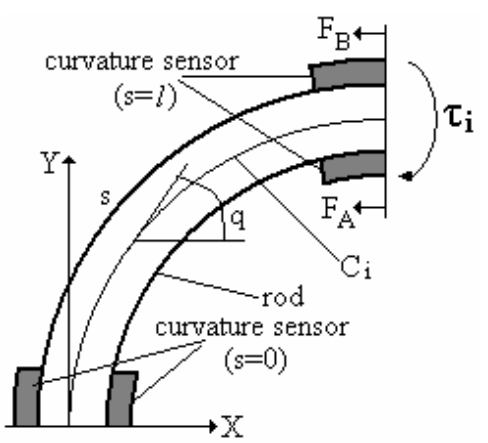

Figure 2. A hyper-redundant segment 
The high flexibility is obtained by an elastic non-extensible backbone rod with distributed damping and negligible shear effects. The driving system consists by two antagonistic cable actuators that are connected at the end of each segment and determine the bending of the arm. The position measuring of the segment is obtained by an electro-active polymer curvature sensor that is placed on the surface at the terminal sub-regions of each segment. These sensors can measure the curvature on the boundary of the segment $(s=0$ or $s=l)$.

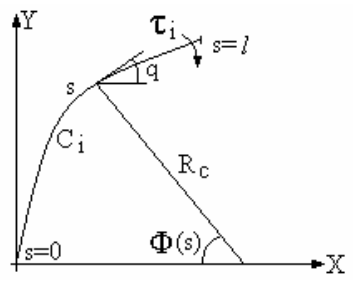

Figure 3. The hyper-redundant arm parameters

The essence of the segment $i$ is the backbone curve $C_{i}$. The length of each segment is $l$. The independent parameter $s$ is related to the arclength from origin of the curve $C_{i}, s \in \Omega$, $\Omega=[0, l][15]$ (Figure 3 ). We denote by $\tau$ the equivalent moment at the end of the segment $(s=l)$ exercised by the cable forces $F_{A}$ and $F_{B}$. The position of a point $\mathrm{s}$ on curve $C_{i}$ is defined by the position vector $r=r(s)$, $s \in[0, l]$. For a dynamic motion, the time variable will be introduced, $r=r(s, t)$. The segment has the elastic modules $E$, the moment of inertia $I$, the bending stiffness $E I$, the linear mass density $\rho$ and rotational inertial density $I_{\rho}$.

\section{Dynamic model}

The dynamic model of a segment can be derived from the Hamiltonian principle. Using the same procedure as in [3] yields the partial differential equations of the arm segment,

$$
I_{\rho} \ddot{q}+b_{1} \dot{q}-E I q_{s s}+c_{1} q=0
$$

with the initial and boundary conditions

$$
\begin{aligned}
& \dot{q}(0, s)=0 \\
& E I q_{s}(t, l)=\tau \\
& q_{s}(t, 0)=0
\end{aligned}
$$

where $q=q(t, s), \dot{q}, q_{s}, q_{s s}$ denote $\frac{\partial q(t, s)}{\partial t}$, $\frac{\partial q(t, s)}{\partial s}, \frac{\partial^{2} q(t, s)}{\partial s^{2}}$, respectively, $b_{1}$ is the equivalent damping coefficient and $c_{1}$ characterizes the elastic behavior.

The equations (3.1) - (3.4) can be rewritten as

$\ddot{q}=a q_{s s}+b \dot{q}+c q$

$q_{s}(t, 0)=0$

$q_{s}(t, l)=d \cdot \tau$

$\dot{q}(0, s)=0, s \in[0, l]$

where

$a=\frac{E I}{I_{\rho}} ; b=-\frac{b_{1}}{I_{\rho}} ; c=-\frac{c_{1}}{I_{\rho}} ; d=\frac{1}{E I}$

The input of the system is represented by the moment $\tau$ applied at the boundary $s=l$ of the arm. The output is determined by the angle values measured by the sensor,

$$
y(t)=q(0, t)
$$

or

$$
y(t)=q(l, t)
$$

\section{Control}

Standard feedback control design assumes fullstate feedback with measurement of the entire state. In this case, the placement of the film sensors at the boundary sub-regions of the arm segment requires designing the state observer.

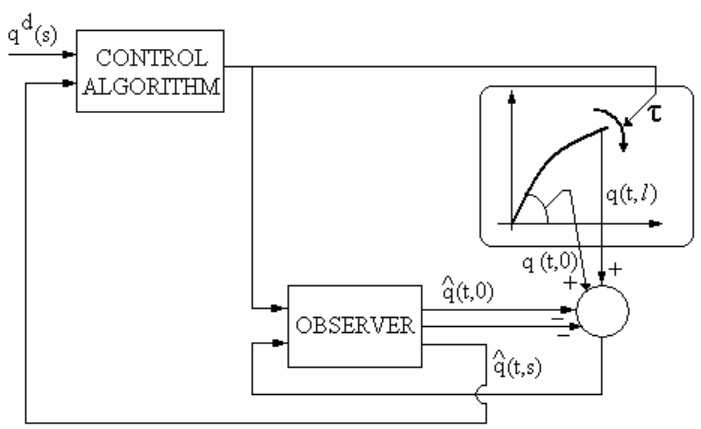

Figure 4. Control system

The following observer is proposed 


$$
\begin{aligned}
& \ddot{\hat{q}}=a \hat{q}_{s s}+b \dot{\hat{q}}+c \hat{q}+ \\
& +k_{10}(s)(q(t, 0)-\hat{q}(t, 0))+ \\
& +k_{11}(s)(q(t, l)-\hat{q}(t, l)) \\
& \hat{q}_{s}(t, 0)=k_{00}(q(t, 0)-\hat{q}(t, 0)) \\
& \hat{q}_{s}(t, 0)=k_{01}(q(t, l)-\hat{q}(t, l))+d \cdot \tau \\
& \dot{\hat{q}}(0,0)=0 \\
& \dot{\hat{q}}(0, l)=0
\end{aligned}
$$

where $\hat{q}=\hat{q}(t, s)$ is the observer state and $k_{10}(s), k_{11}(s), k_{00}, k_{01}$ are the functions and the constants that define the observer parameters. The objective is to determine these parameters in order to reconstruct the state in the domain.

An error variable $\tilde{q}$ is introduced

$$
\tilde{q}=q-\hat{q}
$$

and the error system will be

$$
\begin{aligned}
& \ddot{\tilde{q}}=a \tilde{q}_{s s}+b \dot{\tilde{q}}+c \tilde{q}-k_{10}(s) \tilde{q}(t, 0)- \\
& \quad-k_{11}(s) \tilde{q}(t, l) \\
& \tilde{q}_{s}(t, 0)=-k_{00} \tilde{q}(t, 0) \\
& \tilde{q}_{s}(t, l)=-k_{01} \tilde{q}(t, l) \\
& \dot{\tilde{q}}(0,0)=0 \\
& \dot{\tilde{q}}(0, l)=0
\end{aligned}
$$

where

$$
\lim _{t \rightarrow \infty} \tilde{q}(t, s)=0, s \in[0, l]
$$

We consider that the desired states of the arm motion are given by the curve $C_{d}$,

$$
C_{d}:\left(q^{d}(s), \quad s \in[0, l]\right)
$$

The control problem is to find the moment control law $\tau(t)$ in order to achieve the desired state.

Control algorithm. The closed loop control law of the arm (3.5) - (3.8) with the boundary observer (4.1) - (4.5) is given by

$$
\tau=-\frac{1}{d}\left(k^{1}(l, l)-k^{0}(l, l)\right)\left(q(t, l)-q^{d}(l)\right)
$$

where $k^{1}(s, z), k^{0}(s, z)$ are the solutions of the following partial differential equations,

$$
\begin{aligned}
& a k_{s s}^{1}-a k_{z z}^{1}-c k^{1}=0 \\
& a k_{s s}^{0}-a k_{z z}^{0}-c k^{0}=0 \\
& \frac{d}{d s} k^{1}(s, s)=\frac{c}{4 a} \\
& \frac{d}{d s} k^{0}(s, s)=-\frac{c}{4 a}
\end{aligned}
$$

with the boundary conditions

$$
\begin{aligned}
& k^{1}(0, z)=0, k^{0}(l, z)=0, z \in[0, l] \\
& k_{s}^{0}(l, z)=0, k_{s}^{1}(0, z)=0, z \in[0, l]
\end{aligned}
$$

and the observer parameters are defined by the equations

$$
\begin{aligned}
& k_{01}=k^{1}(l, l)-k^{0}(l, l) \\
& k_{00}=k^{1}(0,0)-k^{0}(0,0) \\
& -k_{10}(s)+\int_{s}^{l} k^{1}(s, z) k_{10}(z) d z+ \\
& \quad+\int_{0}^{s} k^{0}(s, z) k_{10}(z) d z-a k_{z}^{0}(s, 0)=0 \\
& -k_{11}(s)+\int_{s}^{l} k^{1}(s, z) k_{11}(z) d z+ \\
& \quad+\int_{0}^{s} k^{0}(s, z) k_{11}(z) d z+a k_{z}^{1}(s, l)=0
\end{aligned}
$$

Proof. See Appendix.

\section{Conclusions}

The paper treats the control problem of a class of hyper-redundant arms with continuum elements. The observability problems for these models described by partial differential equations are analyzed. The measuring systems are based on the sensors placed on the boundary of the arm. Several observers are proposed for reconstructing the full state of the arm. A back-stepping technique is used in order to design a boundary control algorithm.

\section{Acknowledgement}

The research presented in this paper was supported by the Romanian National University Research Council CNCSIS 
through the IDEI Research Grant ID93 and by CNCSIS - UEFISCSU, project number PNII - IDEI code 289/2008.

\section{Appendix}

The control algorithm is derived by using the back-stepping method developed in $[13,14]$. The coordinate transformation

$$
\begin{aligned}
w(t, s) & =\tilde{q}(t, s)-\int_{0}^{s} k^{0}(s, z) \tilde{q}(t, z) d z- \\
& -\int_{s}^{l} k^{1}(s, z) \tilde{q}(t, z) d z
\end{aligned}
$$

transforms the error system (4.7) - (4.11) into a stable $(b<0)$ target system

$$
\begin{aligned}
& \ddot{w}=a w_{s s}+b \dot{w} \\
& w_{s}(t, 0)=0 \\
& w_{s}(t, l)=0 \\
& \dot{w}(0, s)=0, s \in\left[\begin{array}{ll}
0, & l
\end{array}\right]
\end{aligned}
$$

where

$$
\lim _{t \rightarrow \infty} w(t, s)=0, s \in[0, l]
$$

From (A.1) we obtain

$$
\begin{aligned}
\ddot{w}= & \ddot{\tilde{q}}-\int_{0}^{s} k^{0}(s, z) \ddot{\tilde{q}}(t, z) d z- \\
& -\int_{s}^{l} k^{1}(s, z) \ddot{\tilde{q}}(t, z) d z \\
w_{s s}= & \tilde{q}_{s s}-\int_{s}^{l} k_{s s}^{1}(s, z) \tilde{q}(t, z) d z-k_{s}^{1}(s, s) \tilde{q}(t, s) \\
& +k^{1}(s, s) \tilde{q}_{s}(t, s)+\frac{d}{d s} k^{1}(s, s) \tilde{q}_{s}(t, s) \\
& -\int_{s}^{l} k_{s s}^{0}(s, z) \tilde{q}(t, z) d z-k_{s}^{0}(s, s) \tilde{q}(t, s)- \\
& -\frac{d}{d s} k^{0}(s, s) \tilde{q}(t, s)-k^{0}(s, s) \tilde{q}_{s}(t, s)
\end{aligned}
$$

If we substitute (4.7), (A.7) and (A.8) in (A.2) and integrate by parts, we get the conditions (4.15) - (4.18) and (4.21), (4.22). From (A.1), the velocities at $t=0, s=0$, $s=l$ will be

$$
\dot{w}(0,0)=\dot{\tilde{q}}(0,0)-\int_{0}^{l} k^{1}(0, z) \dot{\tilde{q}}(0, z) d z
$$

$\dot{w}(0, l)=\dot{\tilde{q}}(0, l)-\int_{0}^{l} k^{1}(l, z) \dot{\tilde{q}}(l, z) d z$

and by the boundary and initial conditions (4.10), (4.11), (A.5), we obtain

$$
k^{1}(0, z)=0 ; k^{0}(l, z)=0 ; z \in[0, l]
$$

and by using the boundary conditions (4.8), (4.9), for $s=0, s=l$, respectively, the observer parameters $k_{00}, k_{01}$ are obtained.

Also, if we consider that the desired position is defined by $q^{\mathrm{d}}(s), s \in[0, l]$ and use the boundary conditions (4.3) in (A.1), the control law (4.13) is easily obtained.

\section{REFERENCES}

1. HEMAMI, A., Design of Light Weight Flexible Robot Arm, Robots 8 Conference Proceedings, Detroit, USA, June 1984, pp. 1623-1640.

2. GRAVAGNE, IAN A., IAN D. WALKER, On the Kinematics of Remotely - Actuated Continuum Robots, Proc. 2000 IEEE International Conference on Robotics and Automation, San Francisco, CA, April 2000, pp. 2544-2550.

3. GRAVAGNE, IAN A., WALKER, IAN D., Kinematic Transformations for Remotely-Actuated Planar Continuum Robots, Proceedings of 2000 IEEE International Conference on Robotics and Automation, San Francisco, CA, April 2000, pp. 19-26.

4. CHIRIKJIAN, G. S., J. W. BURDICK, An Obstacle Avoidance Algorithm for Hyper-redundant Manipulators, Proceedings of IEEE International Conference on Robotics and Automation. Cincinnati, Ohio, May 1990, pp. 625-631.

5. MOCHIYAMA, H., H. KOBAYASHI, , The Shape Jacobian of a Manipulator with Hyper Degrees of Freedom, Proceedings of 1999 IEEE International Conference on Robotics and Automation, Detroit, May 1999, pp. 2837-2842.

6. ROBINSON, G., J. B. C. DAVIES, Continuum Robots - a State of the Art, Proceedings of 1999 IEEE International 
Conference on Robotics and Automation, Detroit, May 1999, pp. 2849-2854.

7. IVANESCU, M., V. STOIAN, A Variable Structure Controller for a Tentacle Manipulator, Proceedings of IEEE International Conference on Robotics and Automation, Nagoya, Japan, 1995, pp. 3155-3160.

8. IVANESCU, M., M. C. FLORESCU, N. POPESCU, D. POPESCU, Position and Force Control of the Grasping Function for a Hyper-redundant Arm, Proceedings of IEEE International Conference on Robotics and Automation, Pasadena, CA, 2008, pp. 2599-2604.

9. IVANESCU, M., N. BIZDOACA, M. FLORESCU, N. POPESCU, D. POPESCU, Frequency Criteria for the Grasping Control of a Hyperredundant Robot, Proceedings of IEEE International Conference on Robotics and Automation, Anchorage, Alaska (ICRA 2010), May 2010, pp. 1542-1549.

10. IVANESCU, M., M. FLORESCU, N. POPESCU, D. POPESCU, The Control of the Hyper-redundant Manipulator by Frequency Criteria, in rev. Studies in Informatics and Control, ISSN 12201766, vol. 18 issue 3, Sept. 2009, pp. 279-288.
11. IVANESCU, M., M. FLORESCU, N. POPESCU, D. POPESCU, A Compliance Control of a Hyperredundant Robot, in rev. Studies in Informatics and Control, ISSN 12201766, vol.17, issue 2, June 2008, pp. 189-200.

12. MILlER, D. W., S. A. COLLINS, S. P. PELTZMAN, Development of Spatially Convolving Sensors for Structural Control Applications, 31st AIAA Structures, Structural Dynamic and Materials Conference, 1992, paper $90-1127$.

13. KRSTIC, M., A. SMYSHLYAEV, Boundary Control of PDEs: A Short Course on Backstepping Design, VCSB, 2006.

14. KRSTIC, M., Compensation of Infinite - Dimensional Actuator and Sensor Dynamic, IEEE Control Systems, vol. 30, issue 1, Feb. 2010, pp. 22-41.

15. CAMARILLO, D., C. MILNE, Mechanics Modeling of Tendon Driven Continuum Manipulators, IEEE Transaction on Robotics, vol. 24, issue 6, Dec. 2008, pp. 1262-1273. 
